\title{
Wann Endoskopie bei oberer gastrointestinaler Blutung?
}

Bei Patienten mit einer oberen gastrointestinalen Blutung ist immer eine Endoskopie erforderlich. Doch die Frage, ob die Gastroskopie immer sofort durchgeführt werden sollte, wird kontrovers diskutiert. Jetzt konnte erneut eine klinische Studie zeigen, dass die Endoskopie innerhalb von 24 Stunden ausreicht.

- Im Rahmen dieser Studie wurden Patienten mit einer oberen gastrointestinalen Blutung im Hinblick auf die Durchführung der Endoskopie in drei Gruppen eingeteilt, nämlich innerhalb von sechs Stunden, 6-24 Stunden und mehr als 24 Stunden. Die Mortalität, die Notwendigkeit einer Operation und die Zahl der Bluttransfusionen wurde dann innerhalb dieser drei Gruppen verglichen.

Bei 502 Patienten wurde der Verdacht auf eine obere gastrointestinale
Blutung geäußert, und bei 375 Patienten bestand offensichtlich eine solche, die nicht durch Varizen bedingt war. Patienten, die innerhalb von sechs Stunden endoskopiert wurden, unterschieden sich im weiteren Verlauf nicht signifikant von denjenigen,

\section{Kommentar}

Sicherlich kann diese Studie nicht dahingehend interpretiert werden, dass bei allen Patienten mit einer oberen gastrointestinalen Blutung nicht sofort endoskopiert werden muss. Im klinischen Alltag muss die Dringlichkeit der Endoskopie vom klinischen Status abhängig gemacht werden. Bei Verdacht auf eine weiterbestehende stärkere Blutung sollte die Endoskopie so früh wie möglich durchgeführt werden, auch um Bluttransfusionen zu sparen. Ist der Patient jedoch kreislaufstabil, und ergeben sich keine eindeutigen die zwischen 6 und 24 Stunden nach der stationären Aufnahme der Endoskopie zugeführt wurden. Sogar bei Patienten, die eine endoskopische Blutstillung erhielten, war die Zeit bis zur Endoskopie nicht mit einer besseren Prognose assoziiert.

Hinweise für eine stärker weiterbestehende Blutung, so genügt es, die Endoskopie für den nächsten Morgen zu planen, wenn der Patient in den Nachtstunden stationär aufgenommen wird. Auch bei diesem Problem besteht die ärztliche Kunst darin, möglichst frühzeitig die richtige Risikostratifizierung vorzunehmen.

P. Stiefelhagen =

- N. Sarin et al.

Time to endoscopy and outcomes in upper gastrointestinal bleeding Can. J. Gastroenterol. 23 (2009) 489-493

\section{Ein Bild sagt mehr als 1000 Worte}

— In vielen Ländern gibt es teledermatologische Dienste, die eingesandte Fotografien von Hausärzten und anderen Nichtdermatologen befunden. Dieser Hautbefund aus dem Bereich des oberen Rückens einer $\mathrm{Pa}$ tientin wurde an einen derartigen Dienst übersandt, der die Diagnose eines Ecthyma gangraenosum stellte.

Die Diagnose wurde bei der tatsächlichen Vorstellung des Patienten in der Ambulanz bestätigt. Das Ekthyma weist eine derart charakteristische Ausprägung auf, dass anhand des Bildes eine sichere Diagnosestellung möglich ist. Es handelt sich um eine Vaskulitis als Reaktion auf eine Infektion. Im vorliegenden Fall war die Ursache wahrscheinlich eine direkte Inokulation mit metizillinre- sistenten Staphylococcus aureus durch Exkoriation mit den Fingernägeln. Unter der Therapie mit Linezolid, Mupirocin-Salbe, Débridement und Okklusivverbänden heilten die Hautveränderungen vollständig ab.

\section{Kommentar}

Telemedizinische Anwendungen werden noch zu wenig genutzt, dürften aber mit der zunehmenden Digitalisierung von Daten wahrscheinlich ein wichtiges Element der Medizin in den nächsten Jahrzehnten werden. Wenn man bedenkt, wie viel Wege, Wartezeit und organisatorische Pannen damit vermeidbar wären, wundert man sich, warum die Telemedizin nicht rascher Eingang in die Versorgung findet.

H. S. FÜEßL .

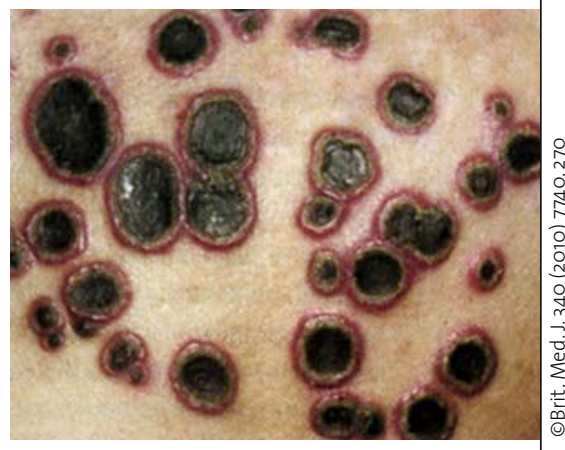

Ecthyma gangraenosum.

- E. V. Smith, R. J. Motley

(Welsh Institute of Dermatology, Glamorgan House, University Hospital Wales, Health Park, Cardiff CF14 4XW, e-mail: dremmasmith@doctors.org.uk). Brit. Med.J. 340 (2010) 7740, 270 